\title{
Nanofluidics Drives Point-of-care Technology for on the Spot Protein Marker Analysis with Rapid Actionable Results
}

\author{
Lauréanne Putallaz, Patrick van den Bogaard, Priscille Laub, Fabien Rebeaud*
}

Abionic SA, Route de la Corniche 5, Epalinges, Switzerland

\begin{abstract}
Protein sensing in a nanofluidic environment dramatically shortens immunoassay time-to-result through the combined action of efficient mass transfer and short diffusion distances. Here, we report on a fully automated point-of-care in vitro diagnostic platform based on disposable capsule containing nanofluidic sensors in which fluorescent immunoassays are performed. Performances of the system were established with three model assays for ferritin, immunoglobulin E (IgE) and pancreatic stone protein (PSP). The described system has the typical high capture efficiency of nano-confined spaces combined with forced-flow, which induce a constant maximal concentration gradient on the sensor. Remarkably, analytes are detected in zeptomole quantities from a drop of blood. Dose-response curves show that high precision and accuracy are achieved in the clinically relevant assay ranges. Moreover, accuracy of the system is excellent agreement with laboratory reference method, as illustrated in a method comparison with total $\mathrm{IgE}$ as a model.

While several academic proof-of-concepts have already described the possibility to exploit the properties of fluids at the nanoscale to develop immunoassay, the transition of these models to a product fulfilling requirements for use at the point-of-care in terms of operability, affordability, reliability and analytic performances remains a challenging endeavor. This study demonstrates that nanofluidic-based immunoassays can efficiently quantify protein biomarkers in the femto- and picomolar range within ultra-short assay time, high precision and accuracy on a closed, small, easyto-operate platform.
\end{abstract}

Keywords: Point-of-care technology; Nanofluidics; Biosensors; Immunoassay; In vitro diagnostic (IVD); EDTA; Immunoglobulin E (IgE); Ferritin

\section{INTRODUCTION}

In vitro diagnostic (IVD) tests performed at the point-of-care are increasingly adopted by health care professional worldwide. Indeed, their short total turnaround time enable fast decisionmaking regarding disease diagnosis, monitoring and clinical management compared to tests performed in laboratories [1], This major advantage makes them especially helpful in clinical emergency situations, such as acute heart failure and myocardial infarction, acute respiratory illness and septic shock [2-4]. However, the benefit of accelerating the clinical decision-making process by on-the-spot biomarker measurement and near-patient environmental conditions is true only if technical, analytical and diagnostic performances are similar to the counterpart tests performed in a controlled laboratory environment. Additionally, point-of-care tests (POCT) must be simple enough to be operated by non-laboratory professionals, have limited preanalytical steps (ideally work directly with unprocessed specimen, i.e. a small volume of capillary whole blood), be maintenance-free and provide unambiguous and actionable test results. Affordability and the capacity to operate in various environments, especially those with extreme environmental conditions in term of temperature, relative humidity and dust particle concentration, are further advantages that may contribute to a more global use of such systems $[5,6]$.

Protein diagnostic tests are largely based on well-established immunoassay techniques. Actually, many strategies are investigated worldwide to respond to the high demand to reduce their assay time and complexity. Specifically, a large effort has been focused on microfluidic systems in which different assay designs have demonstrated the possibility to achieve quantification of molecules in relatively short assay time by "forcing" formation of biomolecular interactions $[7,8]$. Although such fluidic systems are able to show novel and advanced proof of concepts, due to their

${ }^{*}$ Correspondence to: Fabien Rebeaud, Abionic SA, Route de la Corniche 5, Epalinges, Switzerland, Tel: +41213533380; E-mail: fabien.rebeaud@abionic.com

Received: September 20, 2019, Accepted: October 09, 2019, Published: October 16, 2019

Citation: Putallaz L, van den Bogaard P, Laub P, Rebeaud F (2019) Nanofluidics drives point-of-care technology for on the spot protein marker analysis with rapid actionable results. J Nanomed Nanotech. 10:536. doi: 10.35248/2157-7439.19.10.536

Copyright: () 2019 Putallaz L, et al. This is an open-access article distributed under the terms of the Creative Commons Attribution License, which permits unrestricted use, distribution, and reproduction in any medium, provided the original author and source are credited. 
complex fluidic infrastructure (made of multiple interconnected tubes, valves, pressure control systems...) and signal detection systems, only few of them have eventually turned into a robust and manufacturable product that fulfil the essential requirements of a commercial POCT product.

Recent progress in material sciences reduced many of the technical challenges imposed by microfluidic systems, advancing the study of fluidic behavior in the nanometric space as well as the improving capacity to manufacture micro/nano features at large scale and low-cost $[9,10]$. Using such systems, several academic works have claimed that heterogeneous immunoassays can reach a near $100 \%$ capture efficiency of target analyte and an increase in reaction rates by improving mass-transfer and imposing nanoconfinement $[11,12]$. These advances offer unprecedented opportunities to develop small sample volumes diagnostic technologies with ultrashort assay times and truly low analytical sensitivity. system can be integrated in a closed, fully automated device fulfilling all the essential requirement of POCT for the quantification of clinically-relevant biomarkers to support immediate clinical management from a drop of capillary whole blood. Binding equilibrium of the enhanced biomolecular interactions is achieved on a scale of minutes for three different immunoassays designed to measure protein biomarkers of various sizes and structures. We also demonstrate that high sensitivity can be achieved and
To the best of our knowledge, we demonstrate that a nanofluidic

that the analytical performances of these assays are meeting the requirements for use in clinical settings by comparing test results with a laboratory reference method.

\section{MATERIALS AND METHODS}

\section{Testing platform and sample measurement procedure}

The microelectromechanical system (MEMS) nanofluidic sensors are produced by standard microfabrication processes, which is very cost-effective as thousands of sensors are produced on a single wafer. The sensing surface present in the nanoscale portion of the sensors is functionalized using a bulk diffusion process to build a biomolecular interface layer to specifically capture the analyte of interest (Figure 1A).

Biofunctionalized sensors are assembled in a capsule holder (Figure 1B). The capsule contains features to retain cells from a blood sample and to equally distribute the filtered plasma to the biosensors, allowing the use of either capillary whole blood or whole blood processed into serum or anticoagulated plasma. The testing procedure simply requires the user to mix the sample with a buffered solution containing the fluorescent detecting antibodies and to deposit this mix onto the dedicated open slot of the capsule. $30 \mathrm{ul}$ of sample is sufficient for one test and results are available within minutes.

B)

A)

$4000 \mu \mathrm{m}$
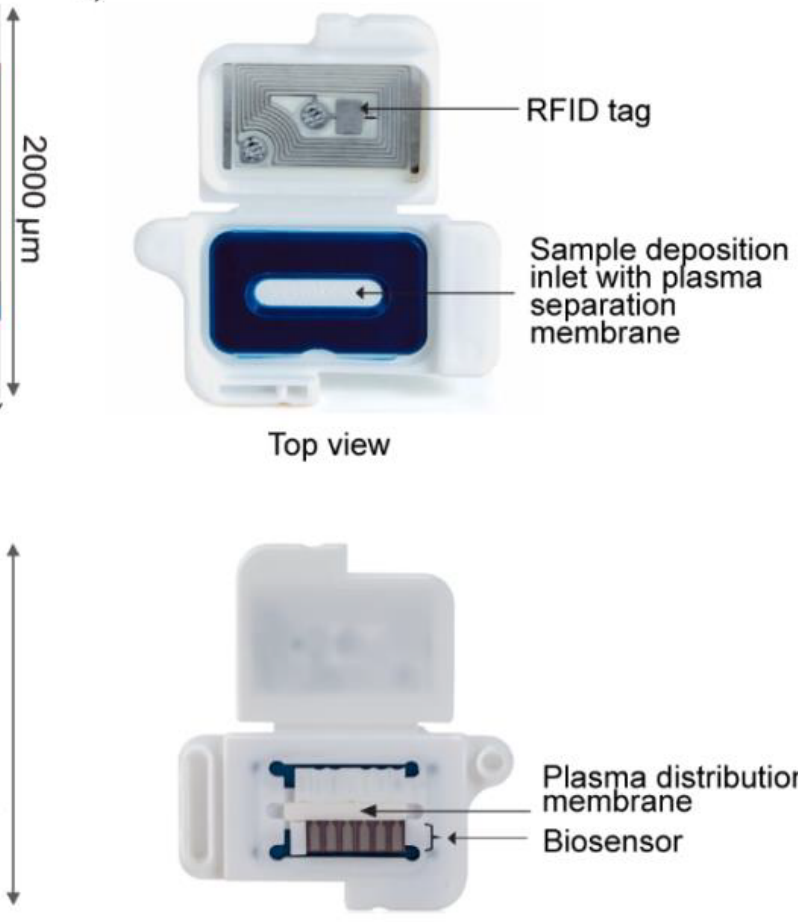

Plasma distribution membrane Biosensor

\section{Other patient proteins \\ Patient analyte \\ Fluorescently labeled detecting antibody \\ - Capture molecule \\ Glass \\ - Adhesion laye}

Figure 1: Fabrication and biofunctionalization of MEMS nanofluidic sensors embedded in a molded plastic injection capsule. (A) Conceptual drawing of the sensors (top panel) showing the microfluidic entry channels (on the left side) and the connected nano-scaled channel (orange area) that is biofunctionalized. The sample flows from the left to the right through passive capillary action triggered by the high surface-to-volume ratio of the hydrophilic reservoir. Magnification on the functionalized nanochannel is also shown (lateral view, bottom panel, not to scale). The sensing surface area is specifically modified to allow immobilization of the capture molecules, while the surrounding surfaces are coated with a hydrophilic polymer to prevent non-specific adsorption of biomolecules. (B) Picture of the top and bottom view of the open capsule holder in which the sensors are embedded, showing the communication (RFID) system to the reading device placed in the capsule lid and the membrane on which the sample is deposited, which filters-out the whole blood cell components and drives by capillarity action the sample to the entry of the biosensors. MEMS: microelectromechanical systems; RFID: Radio-Frequency Identification. 
The device ('abioSCOPE®') is a portable tabletop system that reads the capsules containing the biosensors. The system is comprised of a compact optical measurement unit, a mechatronic system for capsule handling and positioning, an embedded software including data management, connectivity, an interface for human-machine interaction and to display the results. The optical system excites the bound fluorescent complexes with a laser diode and measures the emitted fluorescence intensities on the sensing area. Consecutively, the signals are analyzed in a process which includes assay specific parameters as well as batch calibration information, and finally converted into analyte concentration in the measured sample. The assay and batch parameters (including test identification and measurement protocol, calibration data, batch expiration date and re-use check) are stored in each individual capsule and transmitted to the reader by a radio-frequency identification (RFID) tag contained in the lid.

\section{Kinetic analyte measurement}

Fluorescence intensity of individual biosensors was recorded every 20 seconds for up to 6 minutes by the reading device. Samples were added onto the capsule after the third measurement to precisely assess the initiation of the reaction. A commercially available K3-EDTA venous plasma sample immunodepleted of ferritin (TRINA BIOREACTIVE AG, Naenikon, Switzerland) was further immunodepleted of immunoglobulin of type $\mathrm{E}$ ( $\operatorname{IgE}$ ) and pancreatic stone protein (PSP) by incubating the sample with solid phase-bound anti-IgE, respectively anti-PSP antibodies. With this zero sample material, various analyte doses were generated by spiking in human IgE (preparation 11/234, NIBSC, Potters Bar, United Kingdom), human ferritin (preparation 94/572, NIBSC, Potters Bar, United Kingdom), or a recombinant human PSP (Abionic SA, Epalinges, Switzerland). Each dose was measured in quintuplicate.

\section{Dose-response calibration curves}

Assay performance characteristics were determined by assessing the relationship between doses of analyte with known concentrations and the system response. Dose-response relationships were determined by fitting data points corresponding to the mean value of multiple replicates of calibrator doses using fitting strategies commonly performed in immunoassays, such as four or five parameter logistic model. The selected fitting model was based on the lowest minimized relative error and the backcalculated imprecision for each dose across the clinically relevant concentration range. Calibrators were spiked with either human IgE (preparation 11/234, NIBSC, Potters Bar, United Kingdom), human ferritin (preparation 94/572, NIBSC, Potters Bar, United Kingdom), or a recombinant human PSP (Abionic SA, Epalinges, Switzerland).

\section{Comparison with reference clinical laboratory method}

Total IgE was measured in 69 K3-EDTA anticoagulated whole blood clinical samples in abioSCOPE (Abionic SA, Epalinges, Switzerland). For comparison total $\operatorname{IgE}$ was measured in the corresponding K3-EDTA anticoagulated plasma of the same clinical samples on a Phadia Laboratory System (ThermoFisher Scientific, Uppsala, Sweden), following the product's instruction for use. Agreement between methods was assessed using Deming regression statistics (GraphPad Prism version 8.2.0, GraphPad Software, San Diego, USA).

\section{RESULTS}

The precisely dimensioned sensors comprise a nanoscale fluidic channel connected to micrometer scale lateral openings. Capillary forces drive the entry and constant directional flow of the sample through the nanochannel that contains the sensing surface. A capillary reservoir that holds a fixed volume is located downstream of the nanochannel as depicted in Figure 1A. Capture molecules are specifically immobilized on the sensing area of the nanofluidic channel. Sensors are embedded in a capsule onto which the sample is deposited (Figure 1B). Membranes filter-out the blood cells and drive the resulting plasma mixed with the detecting fluorescent antibodies to the entry of the sensors. Assay parameters such as sample dilution, composition of the assay diluent, concentration of the fluorescent detecting antibody are adapted per test to meet the performance requirements, particularly regarding assay reportable range.

A modified version of the reading device was used to excite the labelled complexes and collect emitted fluorescent signals every 20 seconds for up to 6 minutes from the sensing area of a single sensor per capsule. Signal increase versus time was computed for three different analytes selected for their individual biophysical properties: PSP is a small $(16 \mathrm{kDa})$ monomeric protein, IgE is a large $(190 \mathrm{kDa})$ antibody made-up of two subunits, each present in duplicate (2 heavy chains and 2 light chains), and ferritin is a globular protein complex comprising 24 units of two subunits ("heavy" and "light" forms) present in various ratios, with a total molecular weight of approximately $470 \mathrm{kDa}$. A rapid dose-dependent signal uptake was observed in this system upon deposition of the samples on the capsule, which reached a plateau after approximately two minutes for ferritin (Figure 2A) and PSP (Figure 2B) and after 5 minutes for IgE (Figure 2C). The difference between signal increase rates for the various doses can already be resolved early in the reaction before they reach their plateaus, as is shown for ferritin as an example (Figure 2A). Bleaching of the fluorophores occurring by consecutive excitation is negligible as less than $1 \%$ of signal decrease is generally observed per measurement in independent experiments (data not shown). Capture profiles for the five ferritin doses are depicted in Figure 3 and show a predominant capture at the entry of the nanochannel, with a maximum signal intensity reached in the first 20 pixels of the sensing area followed by a steep decrease in the direction of flow. The overall capture profiles observed are a measure of the analyte concentration and the affinities of the binding partners. Due to optical convolution of the signal, fluorescence proportional to the dose is detected in front of the sensing surface. This is an artifact that is not processed by the signal treatment algorithms as the sensing surface is precisely localized by the reader.

Commonly measured blood protein biomarkers typically cover concentrations in the femtomolar $\left(10^{-15}, \mathrm{fM}\right)$ to the millimolar $\left(10^{-3}\right.$, $\mathrm{mM}$ ) range. Consequently, assay parameters and signal processing must be adapted per tests to ensure the assay reportable range meets the clinical needs. With the aim to demonstrate the capacity of the system to detect truly low protein concentrations in plasma the assay parameters of the ferritin test were further optimized. Figure 4 shows that sub-picomolar concentration of ferritin doses (200, 300 and $500 \mathrm{pg} / \mathrm{ml}$ ) can be reliably distinguished from the zero calibrator (a ferritin-depleted plasma sample) with a dosedependent increasing signal output. The averaged coefficient of variation extracted from the fluorescent output for all doses are below $6 \%$. 

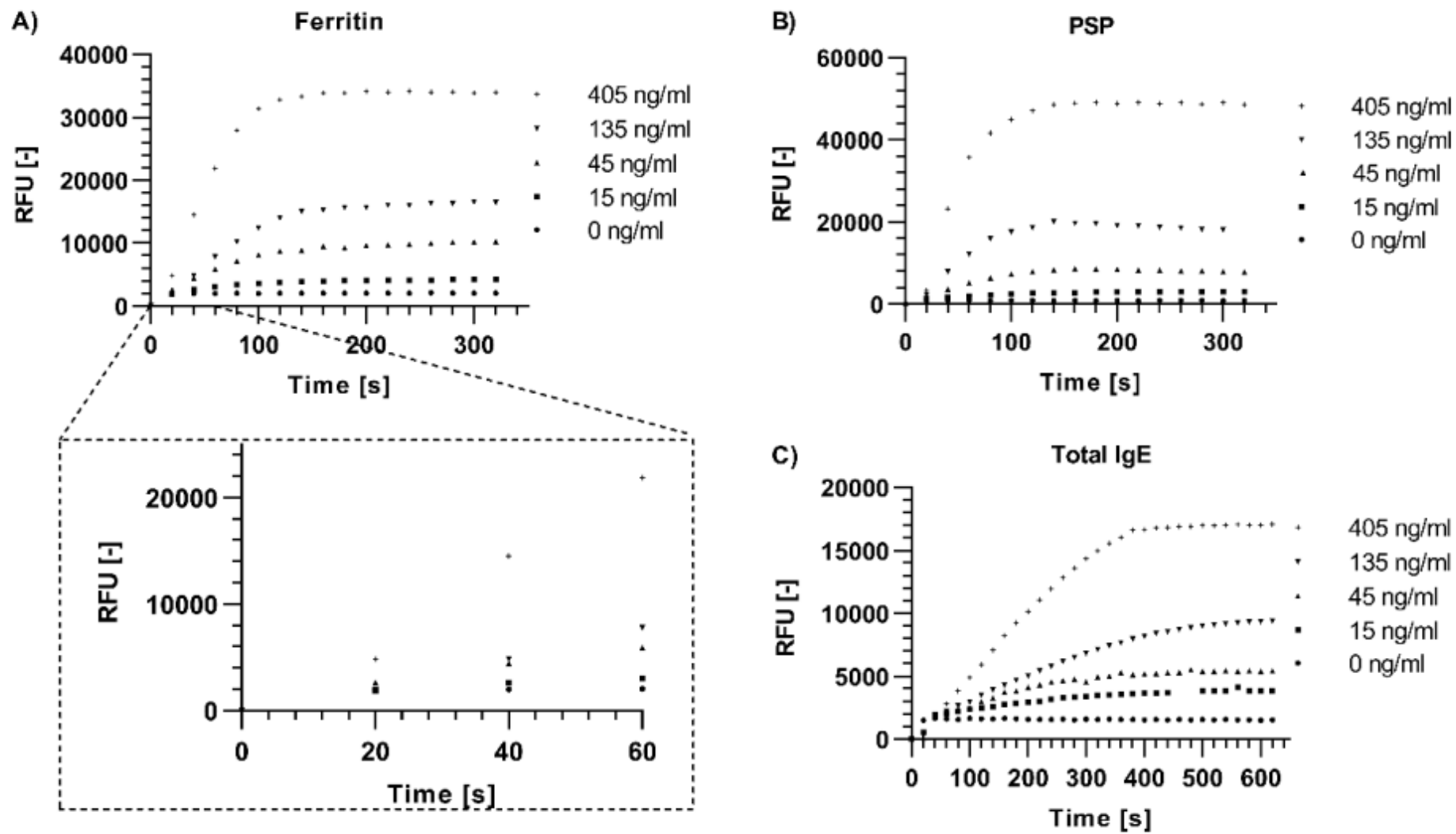

Figure 2: Signal increase in time on the sensing area located in the nanochannel of various analytes in a K3-EDTA anticoagulated plasma matrix. Fluorescence emitted from repeated measurements of the same surface (every 20 seconds for up to 6 minutes) for different doses of ferritin (A), PSP (B) and $\operatorname{IgE}(\mathrm{C})$. Each graph shows one representative sensors from a set of 5 replicates. EDTA: Ethylenediaminetetraacetic acid; IgE: Immunoglobuline of type E; PSP: pancreatic stone protein; RFU: Relative Fluorescence Unit.

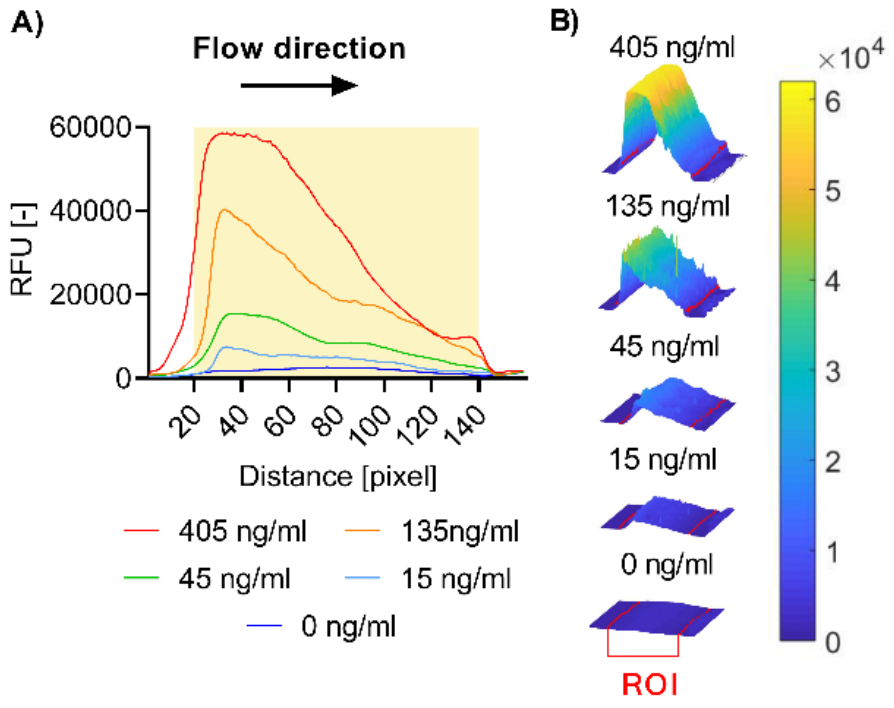

Figure 3: Highly efficient capture of ferritin. (A) Average of the relative fluorescent unit (RFU) value are plotted as a function of the position in the nanochannel, expressed in pixel, for different concentrations of ferritin. The yellow area delineates the boundaries of the sensing area. Data are representative of 5 replicates. (B) RFU profiles as outputted by the reading device. The red lines delineate the ROI, corresponding to the sensing area of the sensor. RFU: Relative Fluorescence Unit.

One of the key requirements for near-patient testing is that test results are sufficiently precise and accurate to ensure patient safety and support that the right clinical decision is taken. Basically, the analytical performances of rapid tests must be in line with what is commonly achieved with reference methods in clinical laboratories. Landmark studies have reported the clinically relevant assay ranges with their corresponding analytical performance

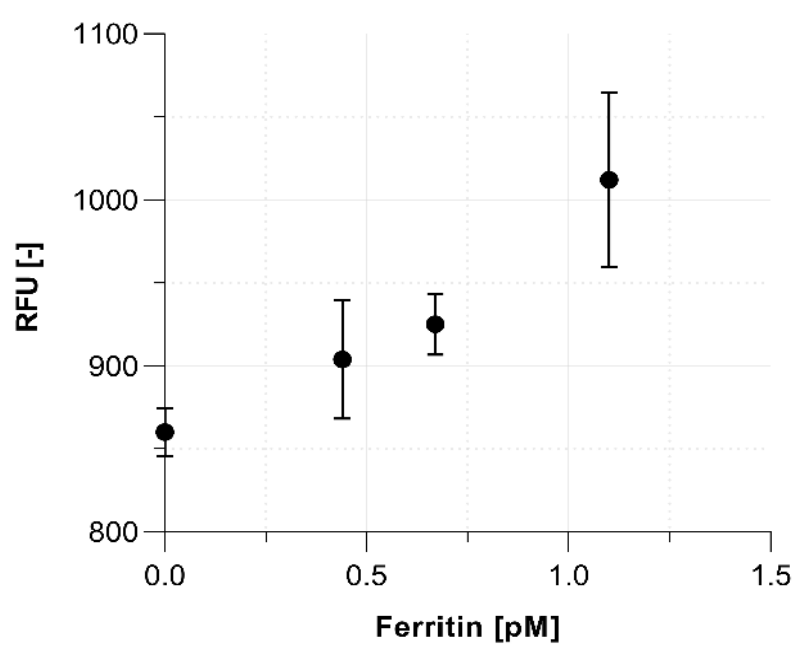

Figure 4: Dose-response curve of ferritin at very low concentration (expressed in picomolar, pM; $1 \mathrm{pM}$ of ferritin corresponds to 474 picogram per milliliter). Average of the relative fluorescent unit (RFU) value are plotted as a function of theoretical concentration, for different concentrations of ferritin. Data are representative of 7 replicates for depleted sample and 3 replicates for other doses.

characteristics for total $\mathrm{IgE}$ to be between 15 and $1500 \mathrm{IU} / \mathrm{mL}$ [13] and for ferritin to be between 12 and $250 \mathrm{ng} / \mathrm{mL}$ [14]. The PSP biomarker range is currently under clinical investigation, but initial internal data suggest it to be $20 \mathrm{ng} / \mathrm{mL}$ to $800 \mathrm{ng} / \mathrm{mL}$ (data not shown). Calibration curves of the three tests show excellent analytical performance characteristics in their respective clinically 
relevant assay range, with all calibrator doses exhibiting a precision below $15 \%$ and an accuracy between $90 \%$ to $110 \%$ (Figure 5).

The performance of the nanofluidics-based IgE immunoassay was finally compared to the gold standard laboratory method. In this method comparison 69 different paired samples (venous K3-EDTA anticoagulated plasma on the reference method and corresponding K3-EDTA anticoagulated whole blood on the nanofluidic system) and the agreement between both methods was assessed using Deming regression statistics. Figure 6 shows that the regression line has a slope of 0.97 with an $95 \%$ confidence interval containing the identity slope of 1 , and an intercept of 0.08 . The two methods having a linear relationship because the null hypothesis was rejected with a $\mathrm{P}$ value $<0.0001$ ) and were therefore judged as significantly comparable.

\section{DISCUSSION}

Immunoassays represent an important part of in vitro diagnostic testing. Today, such assays are still mostly performed in central clinical laboratories on large analyzers. In medical emergency situations or in areas where access to laboratories is cumbersome, point-of-care testing can play a decisive role that is anticipated to continue growing at a steady pace in the coming years [15]. The true value of on-the-spot testing is directly linked to a series of key attributes expected from these novel testing platforms, such as very short time to diagnostic result, minute sample volume, limited or no sample processing and most importantly that the performance characteristics are in line with reference laboratory methods and clinical needs. We have demonstrated here that an innovative system based on nanofluidic sensors integrated into a single use test capsule and a tabletop-size, fully automated reader fulfils indeed all the criteria above.

Firstly, the described sensors, manufactured on wafers from a combination of silicon and glass offer an excellent optical transparency, thermal stability and biological inertness. Moreover, contrary to polydimethylsiloxane (PDMS) based systems, the MEMS scaffold of the biosensors enables mechanical robustness, cost-effectiveness and capacity for large-scale manufacturing using well-controlled processes, which are all essential for a robust commercial product. Secondly, the fluidic system was developed based on a combination of micro- and nanoscale structures and uses only capillary forces instead of active actuation typically used
A)

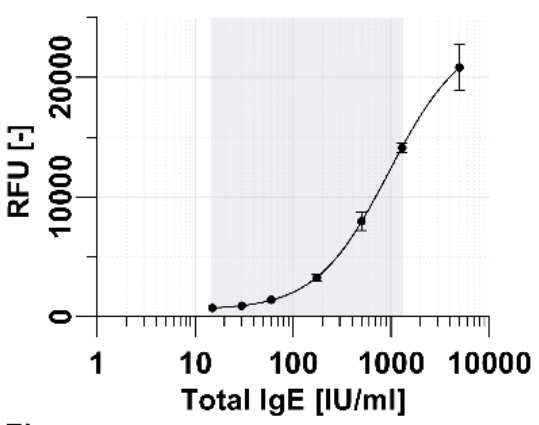

B)

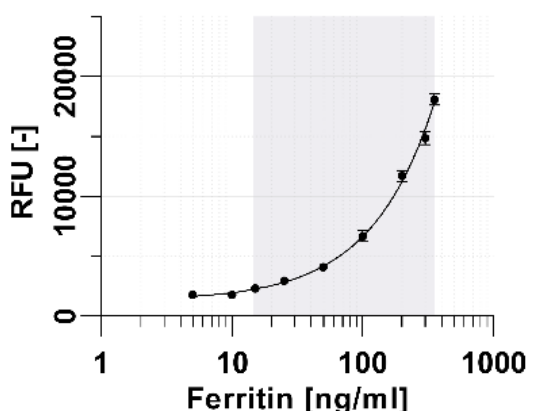

C)

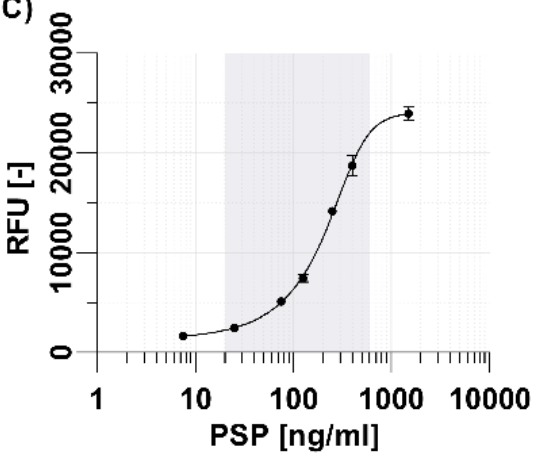

Precision Profile
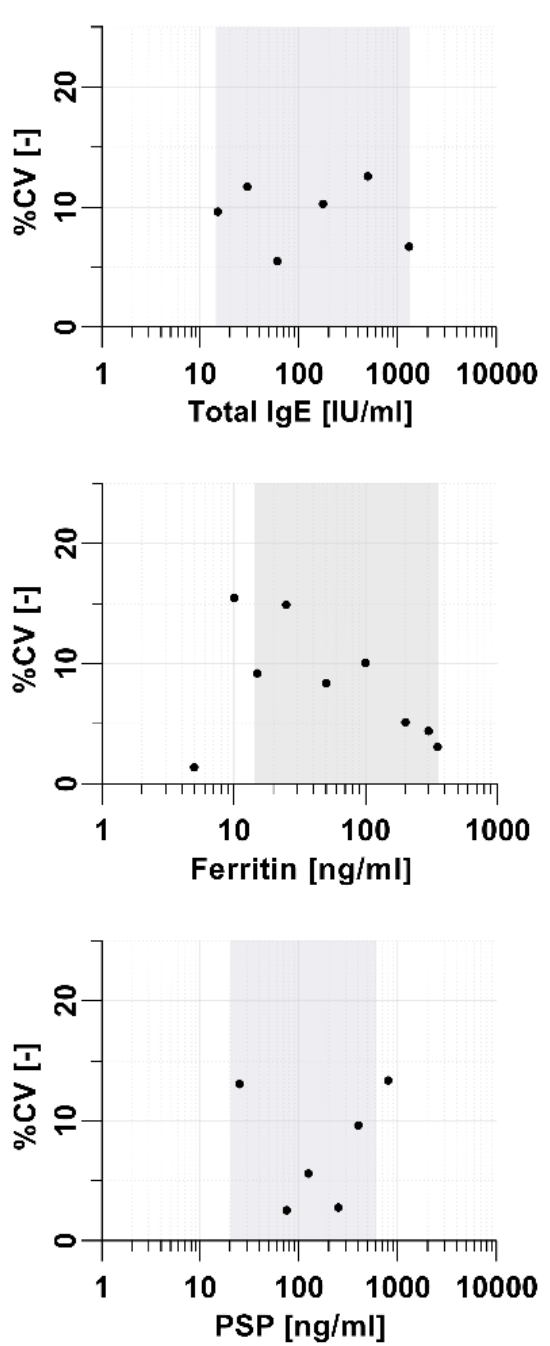
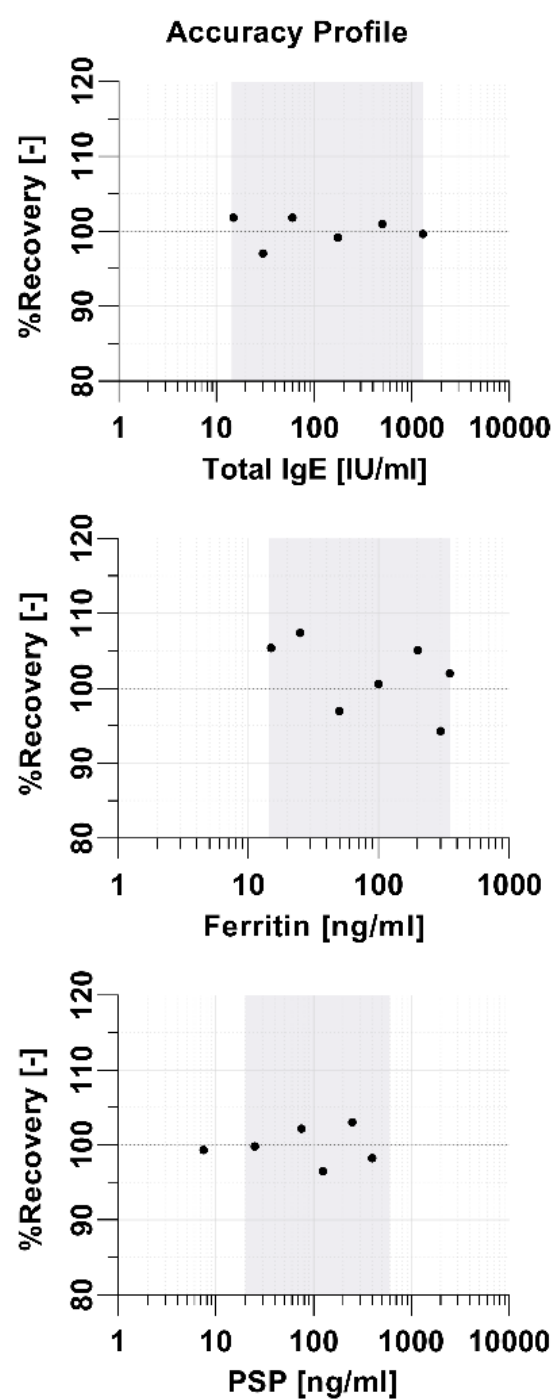

Figure 5: Semi-log graphs representing dose-response calibration curves for $\operatorname{IgE}(\mathrm{A})$, ferritin (B) and PSP (C). Left panels show the fit of the dose responses, central panels show the precision profile expressed in coefficient of variation expressed as percentage (\%CV) for each individual dose, and the right panels show the accuracy profile, expressed in percentages of recovery onto the theoritical value, for each individual dose. Each calibrator dose was measured at minimum 3 times and the data correspond to the mean of the replicates. RFU: Relative Fluorescent Unit, IU/mL: International Unit Per Milliliter, PSP: Pancreatic Stone Protein. 


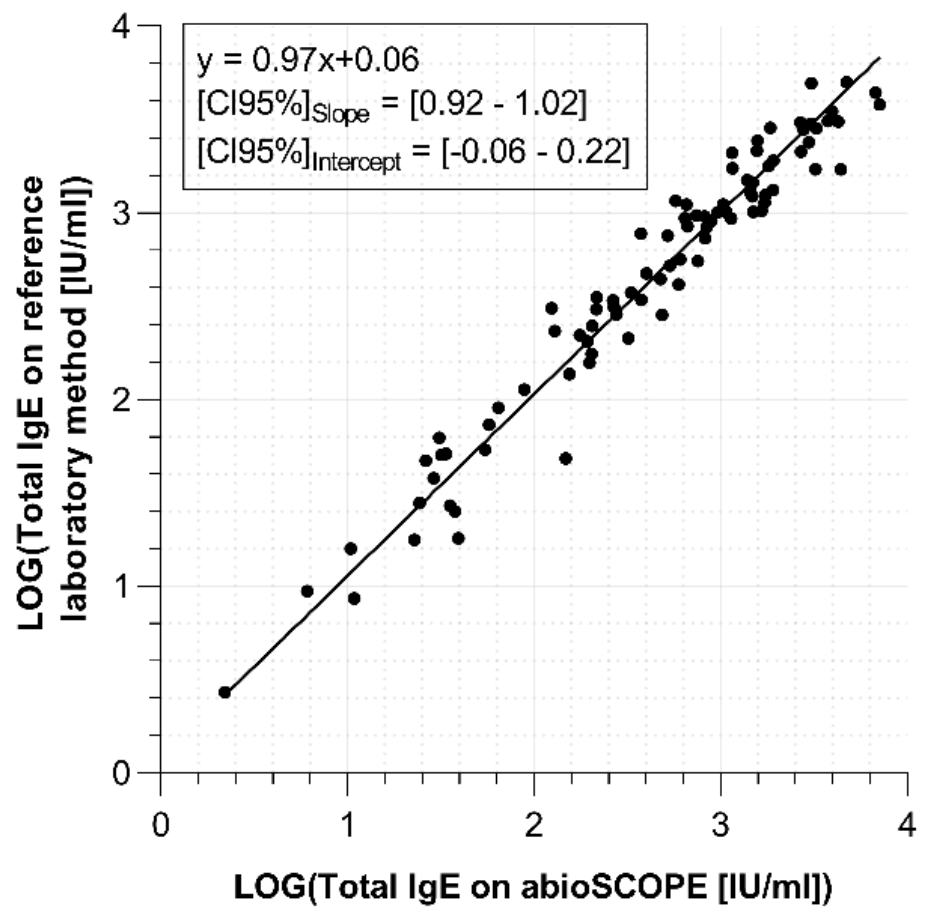

Figure 6: Method comparison of 69 paired samples analyzed as K3-EDTA anticoagulated venous plasma on the laboratory reference method ImmunoCAP ${ }^{\circledR}$ Total IgE (ThermoFisher Scientific, Uppsala, Sweden) and as K3-EDTA anticoagulated whole blood on abioSCOPE ${ }^{\circledR}$ with total IgE nanofluidic immunoassay on abioSCOPE® (Abionic SA, Epalinges, Switzerland). Test results are expressed in IU/mL. Agreement between methods was assessed using Deming regression statistics. Statistical F test on hypothesis of slope being null gave P value <0.0001. EDTA: Ethylenediaminetetraacetic Acid; IgE: Immunoglobulin of Type E; IU/mL: International Unit Per Milliliter.

in the various types of immunoassay analysis systems, thereby greatly reducing the complexity of overall platform. Finally, a smart surface functionalization strategy ensures that the capture molecule is specifically immobilized in the sensing area, i.e. inside the nanochannel, while surrounding surfaces are modified to preclude nonspecific binding.

Several academic works have demonstrated that nanofluidic systems can be used for rapid sensing of biomolecules [16-20] and have provided deep insights, supported by theoretical modelling, into the mechanisms by which biomolecular interactions are enhanced. While differences exist between these systems and the one presented here, notably regarding the fact that we have developed a homogeneous ('no-wash') system without pressuredriven actuation, it is important to point out that the analyte capture behavior observed is in line with the principle of nano-fluidics: efficient mass transfer and nanoconfinement as described in previous studies. In Figure 2, we observed the end of signal increase and the stabilization of the fluorescent values in the minute-range for three completely different analytes and for all concentrations tested. The forced flow combined with nanoconfinement effects lead to ultrashort diffusion distances and impose a constant maximum concentration gradient that forces the reaction equilibrium toward the formation of the final fluorescent complex species thereby reducing regular immunoassay time from several hours to several minutes. The limited volume in combination with increased mass-transfer induces an increased wavefront capture profile in the direction of flow as observed in Figure 3, which is proportional to the concentration of analyte present in the sample. Indeed, for a given assay, the sample flow rate, diffusion coefficient of the analyte and avidities of the binding partners are the same, therefore signal output is purely governed by the analyte concentration. As the sample flow rate and sample volume is constant across assays, the differences in the time needed for each assay to reach an equilibrium is likely to be explained by the avidities of the binding partners. By selecting high affinity capture antibodies for their cognate antigen and specific assay parameters, extremely high ratios between maximum intensity and the width of the capture profile on the sensing surface in the direction of the flow are obtained indicating that the system is able to capture with near $100 \%$ efficiency the analytes, even at very high concentrations (Supplementary Figure 1).

Protein biomarker concentration ranges can be wide and challenges linked to immunoassay-based technology reside in the precise discrimination of low doses. Our system demonstrated a capacity to distinguish ferritin doses in the hundreds of femtomolar concentration from a zero-analytes sample. Translated into a number of analytes passing over the capture area of a sensors, it corresponds to zeptomoles quantities $\left(10^{-21}\right)$. On the other hand, the system can be used to quantify analytes present at high concentration in plasma, such as D-dimers (in the nanomolar range) and C-reactive protein (in the micromolar range) (data not shown). Dose-response calibration curves for the three model proteins, $\operatorname{IgE}$, ferritin and PSP, show that their respective assay ranges, assay repeatability and recovery values are in line with clinical needs. Such results highlight the versatility of the system and its potential for targeting a wide range of clinically relevant analytes.

Analytical performances of point-of-care IVD must be in line with clinical needs so that appropriate medical decision can be immediately taken without jeopardizing patient's safety. In a recently published study, the comparison of two laboratory reference methods have shown a similar agreement for serum total $\operatorname{IgE}$ [21]. Similarly, we have performed a direct comparison of the test results obtained with our nanofluidic system and samples made of whole blood with the corresponding venous plasma samples measured on a laboratory reference method for total IgE further 
highlights that this technology has a very high level of agreement for the diagnostic results.

\section{CONCLUSION}

The success of a POCT system relies on its improvement of the total time to actionable results for a specific analysis. The combination of our robust portable device with the whole blood adapted capsule containing the innovative nano/microfluidic sensors, which we just demonstrated the very short turnover time and reliable performance places our system as a major player for the improvement of critical diagnostics and for the accessibility of health care to everyone.

We report here on the integration of a micro/nanofluidic system in a small reader device designed to quantify proteins on-thespot, hence demonstrating the feasibility of transitioning the nanofluidics research from a fundamental physics-centered stage to an application-oriented stage. Such transition from the bench side to the bedside demands to overcome a series of challenges, such as large-scale, cost-affordable manufacturing of MEMS containing nanofluidic channels, robust surface functionalization processes and test specifications in line with clinical needs. Of particular note, the design of the capsule containing multiple sensors also allows to parallelize testing and thereby providing a multiplex-like design to provide multiple test results from a unique drop of blood.

Nanofluidics have the potential to substantially improve the diagnosis of life-threatening conditions and thereby directly impacting on the chances of survival through the now wellcharacterized fluidic properties and the resulting benefits for rapid formation of biomolecular complexes that can be easily detected by fluorescence or other reporting methods. The technology described here can be further exploited to facilitate access to high quality IVD in primary care settings thanks to its ease of use, small footprint, low installation and maintenance requirements and affordability. It is also possible to extend its utility beyond clinical diagnosis by developing tests for therapeutic drug monitoring or for use as companion diagnosis or to support enrollment and follow up of patient in clinical trials.

\section{ACKNOWLEDGEMENT}

The authors would like to thanks Giulia Crivello, Alix Faillétaz and Yann Sprunger for their excellent technical assistance, as well as Jérome Wagner, Romy Benniga and Olivier Gustarini for all the fruitful discussions and technical support.

\section{REFERENCES}

1. Storrow AB, Zhou C, Gaddis G, Han JH, Miller K, Klubert D, et al. Decreasing Lab Turnaround Time Improves Emergency Department Throughput and Decreases Emergency Medical Services Diversion: A Simulation Model. Acad Emerg Med. 2008;15(11):1130-1135.

2. Kost GJ, Tran NK. Point-of-Care Testing and Cardiac Biomarkers: The Standard of Care and Vision for Chest Pain Centers. Cardiol Clin. 2005;23(4):467-490.

3. https://www.hindawi.com/journals/bmri/2014/731685/

4. Yach D, Hawkes C, Gould CL, Hofman KJ. The Global Burden of Chronic DiseasesOvercoming Impediments to Prevention and Control. JAMA. 2004;291(21):2616-2622.

5. https://onlinelibrary.wiley.com/doi/abs/10.1002/9780470027318. a0540.pub2

6. Drain PK, Hyle EP, Noubary F, Freedberg KA, Wilson D, Bishai WR, et al. Diagnostic point-of-care tests in resource-limited settings. Lancet Infect Dis. 2014;14(3):239-249.

7. Barbosa AI, Reis NM. A critical insight into the development pipeline of microfluidic immunoassay devices for the sensitive quantitation of protein biomarkers at the point of care. Analyst. 2017;142 (6):858-882.

8. Dong J, Ueda H. ELISA-type assays of trace biomarkers using microfluidic methods. Wiley Interdiscip Rev Nanomed Nanobiotechnol. 2017;9(5):e1457.

9. Mijatovic D, Eijkel JCT, van den Berg A. Technologies for nanofluidic systems: top-down vs. bottom-up-a review. Lab Chip. 2005;5(5):492 500 .

10. Mahato K, Maurya PK, Chandra P. Fundamentals and commercial aspects of nanobiosensors in point-of-care clinical diagnostics. 3 Biotech. 2018;8(3):149.

11. Shirai K, Mawatari K, Kitamori T. Extended Nanofluidic Immunochemical Reaction with Femtoliter Sample Volumes. Small. 2014;10(8):1514-1522.

12. Lin Y-L, Huang Y-J, Teerapanich P, Leïchlé T, Chou C-F. Multiplexed immunosensing and kinetics monitoring in nanofluidic devices with highly enhanced target capture efficiency. Biomicrofluidics. 2016;10(3):034114.

13. Gergen PJ, Arbes SJ Jr, Calatroni A, Mitchell HE, Zeldin DC. Total IgE levels and asthma prevalence in the US population: results from the National Health and Nutrition Examination Survey 2005-2006. J Allergy Clin Immunol. 2009;124(3):447-453.

14. https://apps.who.int/iris/handle/10665/85843

15. Fermann GJ, Suyama J. Point of care testing in the emergency department1 1Clinical Laboratory in Emergency Medicine is coordinated by Jonathan S. Olshaker, md, of the University of Maryland Medical Center, Baltimore, Maryland. J Emerg Med. 2002;22(4):393-404.

16. Karnik R, Castelino K, Fan R, Yang P, Majumdar A. Effects of Biological Reactions and Modifications on Conductance of Nanofluidic Channels. Nano Lett. 2005;5(9):1638-1642.

17. Schoch RB, Cheow LF, Han J. Electrical Detection of Fast Reaction Kinetics in Nanochannels with an Induced Flow. Nano Lett. 2007;7(12):3895-3900.

18. Galvin CJ, Shirai K, Rahmani A, Masaya K, Shen AQ. Total Capture, Convection-Limited Nanofluidic Immunoassays Exhibiting Nanoconfinement Effects. Anal Chem. 2018;90(5):3211-3219.

19. Leïchlé T, Chou C-F. Biofunctionalized nanoslits for wash-free and spatially resolved real-time sensing with full target capture. Biomicrofluidics. 2015;9(3):034103-034103.

20. Gervais T, Jensen KF. Mass transport and surface reactions in microfluidic systems. Chem Eng Sci. 2006;61(4):1102-1021.

21. Bulat Lokas S, Plavec D, Rikić Pišković J, Živković J, Nogalo B, Turkalj M, et al. Allergen-Specific IgE Measurement: Intermethod Comparison of Two Assay Systems in Diagnosing Clinical Allergy. J Clin Lab Anal. 2017;31(3):e22047. 